\title{
Hybrid Simulation-Optimization based approach for the Optimal Design of Single-Product Biotechnological Processes
}

\author{
Robert Brunet ${ }^{1}$, Gonzalo Guillén-Gosálbez ${ }^{1 *}$ J. Ricardo Pérez-Correa ${ }^{2}$, \\ José Antonio Caballero ${ }^{3}$ and Laureano Jiménez ${ }^{1}$ \\ ${ }^{1}$ Departament d'Enginyeria Quimica, Escola Tecnica Superior d'Enginyeria Quimica, \\ Universitat Rovira i Virgili, Campus Sescelades, Avinguda Paisos \\ Catalans 26, 43007, Tarragona, Spain \\ ${ }^{2}$ Departamento de Ingenieria Quimica y Bioprocesos, Pontificia Universidad \\ Catolica de Chile, Casilla 306, Correo22, Santiago de Chile, Chile \\ ${ }^{3}$ Departmento de Ingenieria Quimica, Universidad de Alicante, \\ Aparatado de Correos 99. 03080 Alicante, Spain
}

${ }^{*}$ Corresponding author. E-mail: gonzalo.guillen@urv.cat, telephone: +34 977558618 


\begin{abstract}
In this work, we present a systematic method for the optimal development of bioprocesses that relies on the combined use of simulation packages and optimization tools. One of the main advantages of our method is that it allows for the simultaneous optimization of all the individual components of a bioprocess, including the main upstream and downstream units. The design task is mathematically formulated as a mixed-integer dynamic optimization (MIDO) problem, which is solved by a decomposition method that iterates between primal and master sub-problems. The primal dynamic optimization problem optimizes the operating conditions, bioreactor kinetics and equipment sizes, whereas the master levels entails the solution of a tailored mixed-integer linear programming (MILP) model that decides on the values of the integer variables (i.e., number of equipments in parallel and topological decisions). The dynamic optimization primal sub-problems are solved via a sequential approach that integrates the process simulator SuperPro Designer with an external NLP solver implemented in Matlab. The capabilities of the proposed methodology are illustrated through its application to a typical fermentation process and to the production of the amino acid L-lysine.
\end{abstract}

Keywords: hybrid simulation-optimization; mixed-integer dynamic optimization; biotechnological processes; L-lysine. 


\section{INTRODUCTION}

Because of their potential to produce high-value products in human health and care, bioprocesses have recently gained wider interest. The recent boost in competitiveness for customers and new products experienced in this sector has created a clear need for modeling and optimization tools to assist decision-makers in the early stages of the process development.

A bioprocess is a special type of chemical process that produces biochemical products (e.g. antibiotics, proteins, amino acids, etc.) from microorganisms or enzymes. Bioprocesses share some common features with general chemical processes, but differ in their kinetics of product formation, process structure (unit operations and procedures) and operating constraints (सeinzle et an एouba).

Optimization approaches devised so far in biotechnology have primarily focused on the bioreactor step. Cuthrell and Biegler (एप8. optimized a fed-batch reactor for penicillin production with a solution strategy based on successive quadratic programming (SQP) and orthogonal collocation on finite elements. Carrasco and Banga (एप.̣) addressed the dynamic optimization of batch and fed-batch reactors using stochastic optimization algorithms. More recently, Banga et al. (200.5) introduced a new solution method for this problem based on control parameterization, whereas Sarkar and Modalt (200.5) proposed the use of genetic algorithms in this context. For an extensive review of dynamic optimization of bioreactors, the reader is referred to Banga et al. (2003).

Another area related with the bioreactor step that has received attention in the literature is the optimization of metabolic networks. Raghunathan et al. (एण0:3) addressed the data reconciliation and parameter estimation problems in metabolic networks, whereas Guillen Gosalbez and Sorribas (एण0) and Pozo et ad (एण0) have proposed deterministic global optimization techniques for kinetic models of metabolic networks that assist in biotechnological and evolutive studies.

In contrast to these approaches, the optimization of complete bioprocesses considering all their individual steps has received very little attention to date. This can be attributed to 
the fact that these problems lead to complex formulations that integrate structural and operating decisions, some of which change over time. To the best of our knowledge, the work by Groep et al. (2000), is the only one that addressed the optimization of a entire bioprocess (i.e., production of an intracellular enzyme alcohol dehydrogenase). This pioneering work has two main limitations: (i) it assumed a fixed plant topology; and (ii) it applied a simple sensitivity analysis to optimize the operating variables of the process that is not guaranteed to converge to a local (or global) optimum.

Hence, it seems clear that the rich theory available for synthesizing standard chemical process flowsheets has not been applied to the same extent to their biochemical counterparts. In fact, the design of bioprocess flowsheets is nowadays typically accomplished by empirical and/or intuitive methods such as rules of thumb or simple heuristics (एetrides et an एप96), Koulowris et an 2000, Wong et al. 2004 and एetrides et an 2006) that are likely to lead to sub-optimal process alternatives.

With this observation in mind, the aim of this paper is to present a systematic tool for the design of bioprocesses that relies on the combined use of simulation and optimization techniques. More precisely, the design task is formulated as a mixed-integer dynamic optimization (MIDO) problem, which is solved by a hybrid simulation-optimization decomposition method that exploits the complementary strengths of optimization tools (i.e., nonlinear programming, NLP, and mixed-integer linear programming, MILP) and commercial bioprocess simulators (i.e., SuperPro Designer). Our methodology has been tested using two different examples: a typical fermentation process and the production of the amino acid L-Lysine.

\section{PROBLEM STATEMENT}

The problem addressed in this article can be formally stated as follows. Given are the demand and prices of final products, cost parameters, including capital investment and operating cost data (i.e., raw materials and utilities cost), time horizon, thermodynamic properties and per- 
formance models of the equipment units embedded in the flowsheet, including the bioreactor kinetics. The goal of the analysis is to determine the optimal process design, including type and size of process units (e.g., centrifuge, decanter, filtration, etc.), number of equipment units in parallel and operating conditions (concentrations, flow rates, temperatures, etc.) that maximize a given economic performance indicator over a specified time horizon.

In this work, we consider single-product batch plants that can operate with more than one equipment unit (in parallel) per stage. The equipment units in parallel are assumed to be of the same size and operating under the same process conditions. The unit yields are described through nonlinear process models that may require the solution of differential-algebraic equations (DAEs). The operating times and batch sizes are regarded as continuous variables to be optimized rather than as fixed parameters. It should be emphasized that many bioprocesses follow this general pattern, such as the production of penicillin, citric and pyruvic acid, vitamin riboflavin, human serum and insulin, monoclonal antibodies, and plasmid DNA, among many others. It is also important to clarify that in this work the emphasis is on the optimization of the operating conditions and topology of these processes, rather than on the solution of the scheduling problem associated with complex bioprocess batch facilities. The

reader is referred to the review paper by Mendez et ald (2006) for more details on general scheduling approaches, the overwhelming majority of which assume fixed operating times and process yields.

\section{MATHEMATICAL FORMULATION}

Most bioreactors in commercial bioprocesses operate in batch or fed-batch mode. Thus, the reaction kinetics of the biochemical conversions, catalysed either by single enzymes or by cells, are the cornerstones of a bioprocess synthesis problem. The design task requires therefore the simultaneous solution of a mixed-integer non-linear programming (MINLP) synthesis models with embedded DAEs. This gives rise to mixed-integer dynamic optimiza- 
tion (MIDO) problems, the solution of which is a highly difficult task (Bansalet ad [200:3). So far, MIDO algorithms have been applied to the integrated design and control of process plants (Pistikopoulos et al. 2004), simultaneous scheduling and optimal control of reactors (एerrazas-Moreno et an एण07) and optimization of hybrid systems (Allgor and Barton एप9प्). However, to our knowledge, they have never been applied to the optimization of a complete biotechnological process model.

In mathematical terms, the synthesis of biotechnological processes can be posed as a MIDO problem. In this work, we apply the following notation taken from Bansal et ad (एण0):3), which may be simplified in some cases according to the features of the design problem being addressed.

$$
\begin{array}{lll}
\min _{u(t), d, y} & J\left(\dot{x_{d}}\left(t_{f}\right), x_{d}\left(t_{f}\right), x_{a}\left(t_{f}\right), u\left(t_{f}\right), d, y, t_{f}\right) & \\
\text { s.t. } & h_{d}\left(\dot{x_{d}}(t), x_{d}(t), x_{a}(t), u(t), d, y, t\right)=0 & \forall t \in\left[t_{0}, t_{f}\right] \\
& h_{a}\left(x_{d}(t), x_{a}(t), u(t), d, y, t\right)=0 & \forall t \in\left[t_{0}, t_{f}\right] \\
& h_{0}\left(\dot{x_{d}}\left(t_{0}\right), x_{d}\left(t_{0}\right), x_{a}\left(t_{0}\right), u\left(t_{0}\right), d, y, t_{0}\right)=0 & \\
& h_{p}\left(\dot{x_{d}}\left(t_{i}\right), x_{d}\left(t_{i}\right), x_{a}\left(t_{i}\right), u\left(t_{i}\right), d, y, t_{i}\right)=0 & \forall t_{i} \in\left[t_{0}, t_{f}\right] \quad i=1, \ldots, I \\
& g_{p}\left(\dot{x_{d}}\left(t_{i}\right), x_{d}\left(t_{i}\right), x_{a}\left(t_{i}\right), u\left(t_{i}\right), d, y, t_{i}\right) \leq 0 & \forall t_{i} \in\left[t_{0}, t_{f}\right] \quad i=1, \ldots, I \\
& h_{q}(d, y)=0 & \\
& g_{q}(d, y) \leq 0 &
\end{array}
$$

In this formulation, $h_{d}=0$ and $h_{a}=0$ represent the set of differential-algebraic equations (DAEs) that describe the dynamic system whose initial conditions are $h_{0}=0 . h_{p}=0$ and $g_{p} \leq 0$ enforce conditions that must be satisfied at specific time instances, whereas $h_{q}=0$ and $g_{q} \leq 0$ are time invariant equality and inequality constraints, respectively. $x_{d}(t)$ and $x_{a}(t)$ denote the differential state and algebraic variables of the dynamic system, $u(t)$ is the vector of time-varying control variables, $d$ is the vector of time-invariant continuous search variables and $y$ are the binary variables, which in our case are assumed to be time invariant. 
Note that the embedded DAE system is required in order to model the bioreactor kinetics. The binary variables are necessary for representing the different topological decisions, such as the number of equipment units in parallel or the selection of different alternative units in the process. The vector $y$ of binary variables contains $M \cdot N$ components, where $M$ represents the different types of process units and $N$ the maximum number of equipment units in parallel. The component $y_{m, n}$ of this vector takes the value of 1 if $n$ equipment units in parallel of type $m$ are selected, and 0 otherwise. Note that the logical relationships among the binary variables describing connections and interactions between the units in the superstructure are expressed also via constraints $h_{q}=0$.

There are currently two major approaches to solve MIDO problems. The first type relies on converting the MIDO problem into a finite-dimensional MINLP by complete discretization using techniques such as orthogonal collocation on finite elements (Balakrishna and Biegler, [प9:3). The resulting MINLP can then be solved by classical MINLP methods. The second class of MIDO algorithms, to which the strategy presented in this work belongs, is based on the use of reduced space methods (Allgor and Barton, एप9.9). These techniques rely on decomposing the problem into a series of primal dynamic optimization sub-problems with fixed binary variables, and master MILP sub-problems that predict new values of the binary variables for the primal sub-problems.

In complete discretization approaches, the MINLP resulting from the discretization tends to be very large even for relatively small problems, as this approach requires a large number of variables and constraints in order to approximate the solution of the DAE system. On the other hand, in reduced space methods, the difficulty arises in the definition of the master MILP sub-problem. This master problem is typically created by either approximating the nonlinear objective function and constraints by first order linearizations (i.e., supporting hyperplanes) or by deriving Benders cuts from the solution of the primal problem and associated dual information. In the section that follows, we introduce a customized reduced space method for the solution of MIDO problems arising in the design of bioprocesses that 
integrates optimization tools with a bioprocess simulator.

\section{SOLUTION PROCEDURE}

The solution strategy developed in this work is a reduced space method inspired by the

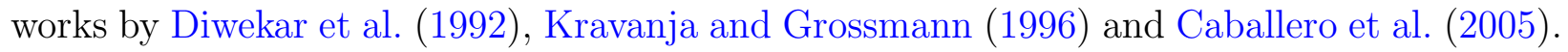
The key ideas of the approach presented are: (i) to integrate mathematical programming tools with a standard bioprocess simulator in the context of a reduced space method for MIDOs; and (ii) to derive a tailored master MILP formulation that exploits the structure of the problem.

The proposed algorithm iterates between master and primal sub-problems, as shown in Figure $\square$. The primal level entails the solution of a dynamic nonlinear programming subproblem, in which the integer decisions, mainly the number of equipments in parallel, are fixed. As discussed in section 5, the solution of this sub-problem requires calculations performed by the bioprocess simulator. On the other hand, the task of the customized master problem is to decide on the value of the integer variables. The algorithm solves iteratively both sub-problems until a termination criterion is satisfied. A stopping criterion that tends to work very well in practice consists of stopping as soon as the primal sub-problems start worsening (i.e. the current primal sub-problem yields an optimal objective function that is worse than the previous one). The main features of these sub-problems are described in detail in the next sub-sections.

(Figure $\square$ could be placed here) 


\subsection{Primal sub-problem}

The primal level entails the solution of a dynamic optimization problem at iteration $k$ of the algorithm for fixed values of the binary variable $k$ :

$$
\begin{aligned}
& \min _{u(t), d, y} J\left(\dot{x_{d}}\left(t_{f}\right), x_{d}\left(t_{f}\right), x_{a}\left(t_{f}\right), u\left(t_{f}\right), d, \bar{y}, t_{f}\right) \\
& \text { s.t. } \quad h_{d}\left(\dot{x_{d}}(t), x_{d}(t), x_{a}(t), u(t), d, \bar{y}, t\right)=0 \quad \forall t \in\left[t_{0}, t_{f}\right] \\
& h_{a}\left(x_{d}(t), x_{a}(t), u(t), d, \bar{y}, t\right)=0 \quad \forall t \in\left[t_{0}, t_{f}\right] \\
& h_{0}\left(\dot{x_{d}}\left(t_{0}\right), x_{d}\left(t_{0}\right), x_{a}\left(t_{0}\right), u\left(t_{0}\right), d, \bar{y}, t_{0}\right)=0 \\
& h_{p}\left(\dot{x_{d}}\left(t_{i}\right), x_{d}\left(t_{i}\right), x_{a}\left(t_{i}\right), u\left(t_{i}\right), d, \bar{y}, t_{i}\right)=0 \quad \forall t_{i} \in\left[t_{0}, t_{f}\right] \quad i=1, \ldots, I \\
& g_{p}\left(\dot{x}_{d}\left(t_{i}\right), x_{d}\left(t_{i}\right), x_{a}\left(t_{i}\right), u\left(t_{i}\right), d, \bar{y}, t_{i}\right) \leq 0 \quad \forall t_{i} \in\left[t_{0}, t_{f}\right] \quad i=1, \ldots, I \\
& h_{q}(d, \bar{y})=0 \\
& g_{q}(d, \bar{y}) \leq 0
\end{aligned}
$$

In the context of our algorithm, this primal sub-problem is solved by parameterizing the control variables, $u(t)$, in terms of time-invariant parameters (reduced space discretisation or control vector parameterisation). Then, for given $u(t)$ and values of the remaining search variables, $d$ (e.g., equipment sizes, operating conditions, etc.) the DAE system is integrated by the process simulator, which in addition to solving the bioreactor kinetics, it calculates mass and energy balances and key economic parameters of the entire process. As will be discussed later on, in some cases it might be necessary to introduce an intermediate module that couples the model implemented in the bioprocess simulator with an external ODE solver algorithm (e.g., implicit Runge-Kutta method). This allows dealing with complex kinetic models that cannot be directly implemented in the process simulator. An external NLP solver is finally employed for searching the values of the control and design variables that maximize the NPV. To accomplish this task, it is necessary to obtain gradient information with respect to the objective function and constraints through finite difference perturbations. 
(Figure $\square$ could be placed here)

Figure $\square$ outlines the solution procedure of the primal sub-problem. One of the main advantages of the approach presented is that it benefits from the unit operations models already implemented in the bioprocess simulator, which avoids having to define them in an explicit form (i.e., equation oriented). This issue facilitates to a large extent the implementation step, as it allows optimizing bioprocess models that are already implemented in the simulator without having to define the associated process and economic equations in an explicit way.

Note that due to the structure of the implicit models in a process simulator, the equations $h_{q}(d, \bar{y})=0$ are eliminated by expressing dependent variables $z$ in terms of decision variables $v$, that is $h_{q}(v, z, \bar{y})=0 \Rightarrow z=\phi_{q}(v)$. Therefore, the NLP subproblem as it arises in a process simulator for fixed binary variables is indeed given as follows:

$$
\begin{array}{lll}
\min _{u(t), d, y} & J\left(\dot{x_{d}}\left(t_{f}\right), x_{d}\left(t_{f}\right), x_{a}\left(t_{f}\right), u\left(t_{f}\right), v, \phi(v), \bar{y}, t_{f}\right) & \\
\text { s.t. } & h_{d}\left(\dot{x_{d}}(t), x_{d}(t), x_{a}(t), u(t), v, \phi(v), \bar{y}, t\right)=0 & \forall t \in\left[t_{0}, t_{f}\right] \\
& h_{a}\left(x_{d}(t), x_{a}(t), u(t), v, \phi(v), \bar{y}, t\right)=0 & \forall t \in\left[t_{0}, t_{f}\right] \\
& h_{0}\left(\dot{x_{d}}\left(t_{0}\right), x_{d}\left(t_{0}\right), x_{a}\left(t_{0}\right), u\left(t_{0}\right), v, \phi(v), \bar{y}, t_{0}\right)=0 & \\
& h_{p}\left(\dot{x_{d}}\left(t_{i}\right), x_{d}\left(t_{i}\right), x_{a}\left(t_{i}\right), u\left(t_{i}\right), v, \phi(v), \bar{y}, t_{i}\right)=0 & \forall t_{i} \in\left[t_{0}, t_{f}\right] \quad i=1, \ldots, I \\
& g_{p}\left(\dot{x_{d}}\left(t_{i}\right), x_{d}\left(t_{i}\right), x_{a}\left(t_{i}\right), u\left(t_{i}\right), v, \phi(v), \bar{y}, t_{i}\right) \leq 0 & \forall t_{i} \in\left[t_{0}, t_{f}\right] \quad i=1, \ldots, I \\
& h_{q}(v, \phi(v), \bar{y})=0 & \\
& g_{q}(v, \phi(v), \bar{y}) \leq 0 &
\end{array}
$$

A very important point in the method is that the process simulator must converge at each time the solver sends a set of values for the design variables. Otherwise the overall procedure will fail. One way to ensure convergence consists of adding slack variables and a penalty to 
the objective function (Viswanathan and Grossmann 15990). This gives rise to the following primal sub-problem:

$$
\begin{aligned}
& \min _{u(t), d, y} J\left(\dot{x_{d}}\left(t_{f}\right), x_{d}\left(t_{f}\right), x_{a}\left(t_{f}\right), u\left(t_{f}\right), v, \phi(v), \bar{y}, t_{f}\right) \\
& +\prod^{T}\left(s_{h p}^{+}+s_{h p}^{-}+s_{g p}+s_{h q}^{+}+s_{h p}^{-}+s_{g q}\right) \\
& \text { s.t. } \quad h_{d}\left(\dot{x_{d}}(t), x_{d}(t), x_{a}(t), u(t), v, \phi(v), \bar{y}, t\right)=0 \\
& \forall t \in\left[t_{0}, t_{f}\right] \\
& h_{a}\left(x_{d}(t), x_{a}(t), u(t), v, \phi(v), \bar{y}, t\right)=0 \\
& \forall t \in\left[t_{0}, t_{f}\right] \\
& h_{0}\left(\dot{x_{d}}\left(t_{0}\right), x_{d}\left(t_{0}\right), x_{a}\left(t_{0}\right), u\left(t_{0}\right), v, \phi(v), \bar{y}, t_{0}\right)=0 \\
& h_{p}\left(\dot{x_{d}}\left(t_{i}\right), x_{d}\left(t_{i}\right), x_{a}\left(t_{i}\right), u\left(t_{i}\right), v, \phi(v), \bar{y}, t_{i}\right)+s_{h p}^{+}-s_{h p}^{-}=0 \\
& \forall t_{i} \in\left[t_{0}, t_{f}\right] \quad i=1, \ldots, I \\
& g_{p}\left(\dot{x_{d}}\left(t_{i}\right), x_{d}\left(t_{i}\right), x_{a}\left(t_{i}\right), u\left(t_{i}\right), v, \phi(v), \bar{y}, t_{i}\right)-s_{g p} \leq 0 \\
& \forall t_{i} \in\left[t_{0}, t_{f}\right] \quad i=1, \ldots, I \\
& h_{q}(v, \phi(v), \bar{y})+s_{h p}^{+}-s_{h p}^{-}=0 \\
& g_{q}(v, \phi(v), \bar{y})-s_{g q} \leq 0
\end{aligned}
$$

where $\prod$ is a penalty parameter vector whose value is finite but chosen to be sufficient large, whereas $s_{h p}^{+}, s_{h p}^{-}, s_{g p}, s_{h q}^{+}, s_{h p}^{-}$and $s_{g q}$ are vectors of positive variables.

\subsection{2. Master sub-problem}

The goal of the master problem is to provide a new set of values for the binary variables that yield better results than the previous one. Here, we present a tailored master MILP that exploits the structure of the problem. Note that due to the presence of nonconvexities in the model, it is not guaranteed that this master MILP will provide a rigorous lower bound on the global optimal solution of the problem.

To generate the master MILP, the design variables are fixed to the optimal value obtained in the latest NLP solved at iteration $k$ of the algorithm, and a series of simulation problems are calculated. The master MILP takes the following form: 


$$
\begin{array}{ll}
\min _{u(t), d, y} & \eta+\Pi^{T}\left(s_{g p}+s_{h q}+s_{g q}\right) \\
\text { s.t. } & \eta \geq J^{k}+\left.\left(\frac{\partial J}{\partial v}\right)\right|_{k}\left(v-\hat{v}^{k}\right)+\left.\sum_{j}\left(\frac{\partial J}{\partial u_{j}}\right)\right|_{k}\left(u_{j}-\hat{u}_{j}^{k}\right)+\Delta J^{k} \cdot y \\
& 0 \geq T_{p}^{k}\left\{h_{p}^{k}+\left.\left(\frac{\partial h_{p}}{\partial v}\right)\right|_{k}\left(v-\hat{v}^{k}\right)+\left.\sum_{j}\left(\frac{\partial h_{p}}{\partial u_{j}}\right)\right|_{k}\left(u_{j}-\hat{u_{j}^{k}}\right)+\Delta h_{p}^{k} \cdot y\right\} \\
s_{g p} \geq g_{p}^{k}+\left.\left(\frac{\partial g_{p}}{\partial v}\right)\right|_{k}\left(v-\hat{v}^{k}\right)+\left.\sum_{j}\left(\frac{\partial g_{p}}{\partial u_{j}}\right)\right|_{k}\left(u_{j}-\hat{u}_{j}^{k}\right)+\Delta g_{p}^{k} \cdot y & \\
s_{h q} \geq T_{q}^{k}\left\{h_{q}^{k}+\left.\left(\frac{\partial h_{q}}{\partial v}\right)\right|_{k}\left(v-\hat{v}^{k}\right)+\Delta h_{q}^{k} \cdot y\right\} \\
s_{g q} \geq g_{q}^{k}+\left.\left(\frac{\partial g_{q}}{\partial v}\right)\right|_{k}\left(v-\hat{v}^{k}\right)+\Delta g_{q}^{k} \cdot y \\
-1 \quad \text { if } \lambda_{p}^{k}<0 \\
0 \quad \text { if } \lambda_{p}^{k}=0 T_{q}^{k}=\left\{\begin{array}{cc}
1 & \text { if } \lambda_{q}^{k}<0 \\
0 & \text { if } \lambda_{q}^{k}=0 \\
1 & \text { if } \lambda_{q}^{k}>0
\end{array}\right.
\end{array}
$$

The objective function is formed by the auxiliary variable $\eta$ and a penalty for constraint violation $\Pi$ that multiplies the slack variables. The linearizations of the objective function and time variant constraints contain three main terms corresponding to: the design variables $\left(\hat{v}^{k}\right)$, control variables $\left(\hat{u}_{j}^{k}\right)$ and the binary variables representing the topological alternatives (y). Note that the control variables $u_{j}$ are parameterized by means of a piecewise constant profile defined on J sub-intervals. On the other hand, the time invariant constraints only consider, the design and topological decisions. In this formulation, $\lambda_{p}^{k}$ and $\lambda_{q}^{k}$ represent the Lagrangean multipliers associated with the time-invariant and time-variant equality constraints, respectively, of the NLP solved at in iteration $k$ of the algorithm. These values are used to correctly relax the equalities into inequalities.

A key issue in this master MILP is how to obtain the derivatives of the objective function and constraints with respect to the decision variables. The derivatives of the continuous variables are approximated by perturbing them in the optimal solution of the NLP problem. 
On the other hand, the partial derivatives with respect to the binary variables, which do not appear explicitly in the MIDO formulation, are determined by running several simulations for different topologies. Note that at each iteration, we need the derivatives of the objective function and the constraints with respect to all the components $y_{m}, n$ of the vector of binary variables. This requires performing at most (depending on the allowable interconections between the equipment units) $M \cdot N-1$ simulations, in each of which we concentrate on changing one single process unit, while keeping the remaining topological decisions fixed. More precisely, we select one process unit $m$ at a time, and run several simulations, each corresponding to a different number $n$ of equipment units in parallel (i.e., from zero, the unit does not exist, up to the maximum number of equipment units in parallel) and leaving the remaining topological decisions unchanged. In performing this step, we discard two types of topological alternatives: (i) those that violate the logical relationships among the binary variables describing allowable connections and interactions between the units, which are expressed via constraints $h q=0$; and (ii) those that are likely to lead to sub-optimal alternatives. To identify topologies of type (ii), we apply a heuristic rule that removes those process alternatives that place equipments in parallel in units others than the bottleneck of the topology found in iteration $k$. Note that all these simulations can be performed very efficiently because the starting point is the optimal solution of the $\mathrm{NLP}^{k}$. Note that to keep the production rate constant in all the simulations, which allows for a fair comparison between the different alternatives being assessed, it is necessary to adjust the input flow rates to the units according to the yields and number of equipment units in parallel. This step can be easily performed with the process simulator assuming that all the process yields remain the same as in the optimal solution of the NLP solved in the previous iteration of the algorithm. It should be noted that all the linear constraints are accumulated in the master MILP, this means that at iteration $k$, the problem includes the constraints generated at the $k^{t h}$ iteration plus all the constraints of all previous iterations. In all cases, after determining the new set of values of the binary variables, the primal problem is solved again, and the overall procedure 
is repeated until the termination criterion is satisfied.

\subsection{Remarks}

- At this point, time varying binary variables are not considered.

- All the required parameters to simulate the bioprocess are initialized in the simulator environment (i.e., properties of the components, equipment parameters, economic parameters, etc.). Also, the number of equipment units in parallel must be specified every time the simulation model is solved.

- The problem addressed in this work can be seen as a special case of the design of single product multi-stage batch plants (see Biegler et an. ए.9.97). Note, however, that in standard scheduling formulations the operating times are assumed to be constant and the process models linear, whereas our approach accounts for variable operating times and nonlinear process models, including the kinetics of the bioreaction.

- Integer cuts can be added to the master problem in order to avoid the repetition of solution explored so far in the primal problem.

- Note that in each iteration of the algorithm we generate linearizations for the process models of both, the existing and non-existing equipment units.

\section{RESULTS}

The capabilities of the proposed approach are illustrated through two case studies. The implementation of the overall method is discussed in first place, whereas the case studies are described next. 


\subsection{Computer architecture / implementation}

The model of the biotechnological plant is developed using SuperPro Designer, (Intelligen, $\mathrm{NJ}$ ), a process simulation tool in which the mass and energy balances as well as the calculation of the key economic indicators are implemented. Note, however, that any other process simulator could be used for the same purpose.

The capabilities of this process simulator are enhanced by coupling it with a dynamic model of the bioreactor coded in Matlab and connected with SuperPro Designer, using the Component Object Module (COM) technology implemented in the Pro-Designer COM Server. The kinetic model of the bioreactor is solved by the odefun function of Matlab. Most of the problems are solved using ode45, which is based on an explicit Runge-Kutta formula, the Dormant-Prince Pair (Forsythe et al. ए977). For stiff problem, the ode15s algorithm (Shampine, [1994) can be employed.

As NLP solvers, we use the fmincon function that implements a sequential quadratic programming (SQP) method. The Hessian of the Lagrangian is updated using the BFGS formula (एowell, [978). The master MILP is implemented in GAMS and solved with CPLEX. The termination criterion applied in the numerical examples is the NLP worsening (i.e., the algorithm stops when the NLPs start to deteriorate). In order to communicate both software packages, we use the interface GAMS-Matlab developed by Ferris (2010).

Note that the function fmincon minimizes a given objective function. In our case, we reverse the sign of the NPV in order to pose the problem as a minimization one.

\subsection{Case Study I. A basic fermentation process}

We first consider a basic illustrative example of a hypothetical fermentation process (Figure 3). The process includes two steps: a reaction that takes place in a fermentor, and a separation that is performed in either a decanter, a centrifuge or a filter. In the reaction-phase, the initial substrate dissolved in water reacts with oxygen forming product A and by-products (waste). In the second stage, the product is separated to obtain pure A. The production 
recipe involves the following operations: charge of water (time neglected), charge of substrate (time neglected), heating (60 min), charge of inoculum (time neglected), fermentation (time to be optimized), cooling (60 min) and transfer out (time neglected) carried out in the reactor; and a separation task whose time depends on the equipment used (decanting 120 min, centrifugation $100 \mathrm{~min}$, and filtration $130 \mathrm{~min})$.

Substrate and water are charged at $25^{\circ} \mathrm{C}$. Then the mixture is heated until the optimal growth temperature (i.e., $37^{\circ} \mathrm{C}$ ) using steam at $152^{\circ} \mathrm{C}$, with an efficiency of $80 \%$ in the heat transfer. The inocolum is added when the optimal temperature is reached. The reaction is an aerobic fermentation carried out at constant temperature that is modeled by a Monod-type kinetics of the following form:

$$
\mu=\mu_{\max } \frac{S}{K_{M}+S}
$$

where $\mu$ is the rate of formation of biomass expressed in $\mathrm{g} / \mathrm{l} \cdot \mathrm{h}, S$ is the concentration of substrate in $\mathrm{g} / \mathrm{l}$ and $K_{M}$ and $\mu_{\max }$ are kinetic parameters that take a value of $0.2 \mathrm{~h}^{-1}$ and 35 $\mathrm{g} / \mathrm{l}$, respectively. The reaction requires an aeration stream of $0.5 \mathrm{VVM}$ (i.e., volume of air per volume of liquid per min). Chilled water is used to remove the metabolic heat (reaction enthalpy equals $-15,478 \mathrm{~kJ} / \mathrm{kg}$ ). The stoichiometry of the reaction is as follows:

$100 \mathrm{~kg}$ Substrate $+70 \mathrm{~kg} \mathrm{O} \mathrm{O}_{2} \rightarrow 28 \mathrm{~kg}$ Biomass $+70 \mathrm{~kg} \mathrm{CO}_{2}+60 \mathrm{~kg} \mathrm{H} \mathrm{H}_{2}+2 \mathrm{~kg} \mathrm{~A}+10$ $\operatorname{kg}$ waste

The final mixture is cooled down to $25^{\circ} \mathrm{C}$, using chilled water and assuming an efficiency of $90 \%$ in the heat transfer. The mixture is then transferred to the separation equipment, where a percentage of $\mathrm{A}$ is separated from the remaining compounds yielding a final product with a purity of $100 \%$. The efficiency in the decanter and filter is $90 \%$ (i.e., $90 \%$ of $\mathrm{A}$ is 
retained and $10 \%$ is lost), whereas that of the centrifuge is $92 \%$.

(Figure [ $\mathrm{B}$ could be placed here)

The design objective is to maximize the NPV assuming a fixed demand of $2025 \mathrm{~kg} / \mathrm{year}$ of A. The entire process is modeled using SuperPro Designer, the kinetic model and the NLP solver are implemented in Matlab, whereas the master MILP is coded in GAMS. The bioreactor is modeled as a stoichiometric reactor in SuperPro Designer, whose conversion is provided by Matlab after integrating the DAE system that describes the kinetic model. The NLP solver is also implemented in Matlab, whereas the master MILP is solved with CPLEX interfacing with GAMS. As NLP solver, we use a gradient based method (i.e., SQP). The continuous decision variables to be optimized are the initial substrate concentration and the reaction time. The integer decision variables represent the number of equipment units in parallel, as well as the selection of a specific separation unit (i.e., decanter, centrifuge and filter) in the downstream section. The NPV calculations are performed with the default parameters used in SuperPro Designer, and assuming that the facility dependent capital costs are zero.

A preliminary analysis of the process is performed prior to the application of the optimization algorithm. Figures fig:figure4a and fig:figure4b show the dependency of the concentration of $\mathrm{A}$ in the reactor and total number of batches with respect to the reaction time and initial substrate concentration for a demand of $2025 \mathrm{~kg} /$ year. As observed, by increasing the reaction time, higher concentrations of A per batch (but fewer number of batches) are obtained. Similarly, increasing the initial substrate concentration leads to higher concentrations of A in each batch. However, as shown in Figure fig:figure5, the completion time of the reaction (i.e., time required to achieve the total depletion of the substrate) increases with the initial substrate concentration. Hence, increasing the substrate concentration indirectly diminishes the total number of batches produced per year. The task of the optimization algorithm is 
therefore to find the proper values of substrate concentration and reaction time and the plant topology that minimize the negative value of the NPV.

(Figure $⿴$ could be placed here)

(Figure 1 could be placed here)

The algorithm is next applied to the problem. It converges after 2 major iterations and 12.46 CPU seconds on a computer AMD Phenom ${ }^{T M}$ 8600B, Triple-Core Processor $2.29 \mathrm{GHz}$ and 3.23 GB of RAM memory.

(Table $\square$ could be placed here)

Table $\square$ shows the starting point and the optimized values obtained. In the base case, the concentration of glucose is $53.47 \mathrm{~g} / \mathrm{l}$, the reaction time is 15.27 hours and only one single equipment is placed in each stage and the separation unit used is the decanter. In the optimized case, the initial concentration of glucose attains its upper bound (i.e. $80 \mathrm{~g} / \mathrm{l}$ ) and the reaction time is 16.18 hours. As observed, the NPV is maximized by increasing the initial substrate concentration up to its upper bound, and by making the reaction time equal to the completion reaction time. By doing so, the water added to the reactor is minimized, and hence its size. This solution does not imply the use of equipment units in parallel either, and the separation unit selected for the optimal design is the decanter. The reason for this is that the decanter is cheaper than the centrifuge (where two units have to be placed in parallel) and the filter. However, for smaller A production rates the centrifuge becomes the best alternative.

With these changes, the NPV is increased by $4.71 \%$ (i.e., from 20,870 M\$ to 21,854 M\$). Note that increasing the initial concentration of substrate leads to larger batch throughputs and lower volumes and capital investment. On the other hand, it also leads to larger cycle 
times, and hence, fewer batches per year. Particularly, in the optimized solution the reactor volume is reduced by $29 \%$ (from 7,675 $\mathrm{l}$ in the base case, to 5,454 $\mathrm{l}$ in the optimal case) and the total capital investment by $4.5 \%$.

(Table $\square$ could be placed here)

\subsection{Case study II. Production of L-lysine}

As second example, we study the production of the amino acid L-Lysine. This product is mainly used as an animal dees additive (for more details the reader is referred to $\mathbb{P f e f f e r l}$ et and 20(0)

This is indeed a more difficult problem that requires the solution of a complex kinetic bioreactor model. The associated flowsheet (see Figure 6) comprises ten major processing units that are aggregated into three different sections: upstream, fermentation and downstream. The upstream processing includes all unit operations required to prepare the feed streams. In this section, the nutrients are mixed in a blending tank and mixed with water before being sterilized and transferred to the fermentor. When the reaction is completed, the mixture is transferred to a stabilization vessel and then filtered. The permeate is pumped to an evaporation unit that removes most of the water content. The broth is finally spray-dried and processed to granules. For biomass removal, we consider the following process alternatives: a rotary vacuum filtration, a micro filtration and a centrifugation.

(Figure $\mathbf{6}$ could be placed here)

The bioprocess includes a fed-batch reactor that uses a genetically modified microorganism (i.e., Corynebacterium glutamicum). The main reactants are threonine, nutrients (glucose, $\mathrm{NH}_{4} \mathrm{OH}$ and $\mathrm{KH}_{2} \mathrm{PO}_{4}$ ), and oxygen. The set of equations describing the reaction kinetics

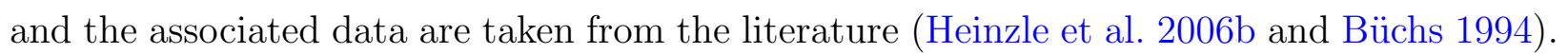


A demand of 6,202 tones Lysine/ year is considered.

The application of our algorithm to this example follows the same implementation scheme discussed in previous sections. Particularly, the bioreactor model that accounts for the kinetics of the lysine production is implemented in Matlab and solved by the ODE solver ode15s. The decision variables are the initial concentrations of threonine and glucose, initial volume of the fermentor (i.e., amount of raw materials fed to the bioreactor) and reaction time, which are the ones with a larger impact on the performance of the process. The discrete variables represent the number of equipment units in parallel and process units used for the biomass removal.

Figures $\square$ and $\mathrm{Q}$ show the results of coupling the bioreactor model with the process model for a fixed topology with one fermentor in parallel and a rotatory vacuum filter. Specifically, in Figure $\square \mathrm{a}$, the unit production cost, the space-time-yield $(S T Y)$ (i.e., mass of L-lysine produced per unit of volume and time in the bioreactor) and the overall yield $\left(Y_{o a}\right)$ (mass of L-lysine produced per mass of glucose consumed) are plotted versus the initial concentration of threonine. In Figure $\square \mathrm{b}$, the same variables are plotted versus the initial concentration of glucose, and in Figures $\mathrm{Ba}$ and $\mathrm{Bb}$ versus the initial fermentor volume and reaction time respectively.

In all cases, we only change one decision variable at a time maintaining the remaining ones constant (1.62 g/l Threonine, $48.72 \mathrm{~g} / \mathrm{l}$ glucose, $310.34 \mathrm{~m}^{3}$ initial fermentor volume and $71.01 \mathrm{~h}$ of reaction time). Let us clarify that all these points violate the demand satisfaction constraint (i.e., production equals the demand of 6,202 tones L-lysine/year).

(Figure $\square$ could be placed here)

(Figure $\square$ could be placed here)

As observed in Figures $\square$ and $\boldsymbol{\nabla}$, the selected variables have a large impact on the bioreactor performance. Within the investigated range of the decision variables, the economical objec- 
tive function is highly dependent on the $S T Y$ and $Y_{o a}$. Note that the economic performance of the process depends to a large extent on the capital investment and operating costs. The former term is mainly influenced by the $S T Y$. Specifically, larger $S T Y$ values lead to lower equipment sizes. On the other hand, the operating costs are mainly affected by the $Y_{\text {oa }}$, since this variable has a large impact on the amount of raw materials consumed.

Higher initial concentrations of threonine increase the $S T Y$, but decrease the $Y_{o a}$. With regard to the glucose, the maximum $S T Y$ and $Y_{\text {oa }}$ are both found at high initial glucose concentration. The initial reaction volume is the decision variable with the smallest effect on the $S T Y$ and $Y_{o a}$. Finally, longer times lead to high values of $S T Y$ and $Y_{o a}$ and lower unit production costs.

We studied the effect of the integer decisions (number of reactors and separators for biomass removal) on the performance of the plant for a given set of initial conditions $(1.62 \mathrm{~g} / \mathrm{l}$ Threonine, $48.72 \mathrm{~g} / \mathrm{l}$ glucose, $310.34 \mathrm{~m} 3$ initial fermentor volume and $71.01 \mathrm{~h}$ of reaction time). Increasing the number of reactor units leads to more batches, and hence smaller equipment units. For biomass removal, three options are presented: a rotary vacuum filter(RVF), a microfilter $(\mathrm{MF})$ and a centrifuge $(\mathrm{CF})$. For the RVF and $\mathrm{MF}$, the operation time is $8 \mathrm{~h}$ and for the centrifuge $6 \mathrm{~h}$. The efficiencies of these units are: $98.5 \%$ (RVF), $93.6 \%(\mathrm{MF})$ and $99.6 \%(\mathrm{CF})$.

The preliminary analysis presented above provides some insight into the problem but cannot lead in itself to optimal solutions. The task of the optimization algorithm is to perform an exhaustive search in the entire parameters space. Two constraints are considered: production less or equal than the demand and a specification on the final concentration of L-lysine (i.e., the mass fraction should be in the range 0.36-0.76 as suggested by Stevens and Bلinder एप.प्प).

The algorithm converged in 4 major iterations and 194.90 CPU seconds on the same computer as before. 
(Table 团 could be placed here)

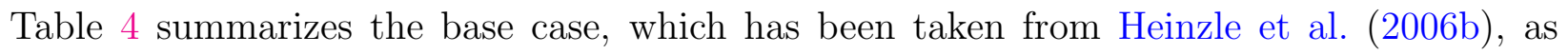
starting point to initialize the overall solution procedure we use this base case solution but considering a different topology (i.e., no reactors in parallel and a rotary vacuum filtration for the biomass removal). As observed, NPV increases by $13.77 \%$ compared to the base case $(195,688 \mathrm{M} \$$ vs. $172,003 \mathrm{M} \$)$. This is accomplished by using two fermentors instead of three (as was the case in the base solution adapted from सeinzle et an 20060) and also by properly adjusting the operating conditions of the fed-batch reactor and the rest of the upstream and downstream equipment capacities. Particularly, in the optimal solution, the initial concentrations of glucose and threonine are higher than in the other cases. These new conditions increase both the $S T Y$ and $Y_{o a}$. The increase in the $S T Y$ leads to a reduction of the equipment sizes and the associated capital investment. On the other hand, increaseing the $Y_{o a}$ reduces the raw materials consumption, and therefore the operating cost. As a result, the total capital investment and operating costs are reduced by $21.5 \%(79.885 \mathrm{M} \$ \mathrm{vs}$ $101.766 \mathrm{M} \$)$ and $16.9 \%(8.830 \mathrm{M} \$ /$ year vs. $10.631 \mathrm{M} \$ /$ year $)$ respectively, while keeping the production rate constants (6,202 tones L-lysine/year).

(Table $⿴$ could be placed here)

\section{Conclusions}

This work has introduced a systematic strategy to assist in the development of biotechnological processes that allows to optimize the operating conditions and topology of the entire bioprocess. The proposed method relies on a reduced space MIDO algorithm that integrates commercial process simulators (SuperPro Designer) with optimization tools (Matlab and GAMS). 
The capabilities of the method presented have been tested in two biotechnological examples: a typical fermentation process, and the production of the amino acid L-lysine. From numerical results, we concluded that it is possible to significantly improve the economic performance of bioprocesses by optimizing them as a whole. Particularly, larger benefits can be attained by properly adjusting the operating conditions and equipment sizes of all the units embedded in the flowsheet. One of the main advantages of our approach is that it makes use of a standard bioprocess simulation package, that implements the main process and economic equations. This largely simplifies the modeling and economic analysis of the whole plant, allowing for the optimization of a wide range of bioprocess facilities.

\section{Acknowledgements}

The authors wish to acknowledge support from the Spanish Ministry of Education and Science (projects DPI2008-04099 and CTQ2009-14420-C02) and the Spanish Ministry of External Affairs (projects A/023551/09, A/031707/10 and HS2007-0006). 


\section{References}

Allgor RJ, Barton PI. Mixed-integer dynamic optimization I: Problem formulation. Computers and Chemical Engineering 1999; 23: 567-584.

Balakrishna S, Biegler LT. A unified approach for the simultaneous synthesis of reaction, energy, and separation systems. Industrial Engineering and Chemical Research 1993; 32:1372-1382.

Banga JR, Moles CG, Balsa-Canto E, Alonso AA. Dynamic optimization of bioreactors-a review. Proc. Ind. Natl. Sci. Acad. 2003; 69:257-265.

Banga JR, Moles CG, Balsa-Canto E, Alonso AA. Dynamic optimization of bioprocesses: Efficient and robust numerical strategies. Journal of Biotechnology 2005; 117:407-419.

Bansal V. Perkins JD, Pistikopoulos EN, Sakizlis V, Ross R. New algorithms for mixedinteger dynamic optimization. Computers and Chemical Engineering 2003; 27:647-668.

Biegler, LT, Grossmann, IE, Westerberg, AW. Systematic methods of chemical process design. Prentice Hall; 1997.

Büchs J, Precise optimization of fermentation processes through integration of bioreaction. Process computations in biotechnology.McGraw-Hill: New Delhi; 1994; 194-237.

Caballero JA, Milan-Yañez D, Grossmann IE. Rigorous design of distillation columns.Ind. Eng. Chem. Res. 2005; 44:6760-6775.

Carrasco E, Banga JR. Dynamic optimization of batch reactors using adaptive stochastic algorithms.Ind. Eng. Chem. Res. 1997; 36:2252-2261.

Cuthrell JE, Biegler LT. Simultaneous optimization and solution methods for batch reactor control profiles. Computers and Chemical Engineering 1989; 13:49-62. 
Diwekar UM, Grossmann IE, Rubin ES. An MINLP Process Synthesizer for a Sequential Modular Simulator. Ind. Eng. Chem. Res. 1992; 31:313-322.

Gill PE., Murray W, Saunders MA. SNOPT: An SQP algorithm for large-scale constrained optimization SIAM Journal on Optimization 2002;12(4):979-1006.

Forsythe G, Malcolm M, Moler C. Computer Methods for Mathematical Computations. Prentice-Hall, New Jersey; 1977.

Groep ME, Gregory ME, Kershenbaum LS, Bogle IDL.. Performance Modeling and Simulation of Biochemical Process Sequences with Interacting Unit Operations.Biotechnology and Bioengineering 2000; 67:300-311.

Guillen-Gosálbez G. Sorribas A. Identifying quantitative operation principles in metabolic pathways: a systematic method for searching feasible enzyme activity patterns leading to cellular adaptive responses. BMC Bioinformatics 2009; 32:1372-1382.

Heinzle E, Biwer AP, Cooney CL. Development of sustainable bioprocesses. modeling and assessment. John Wiley and Sons; 2006; 1-116

Heinzle E, Biwer AP, Cooney CL. Development of sustainable bioprocesses. modeling and assessment. John Wiley and Sons; 2006; 155-165

Kravanja Z, Grossmann I.E. Computational Approach for the Modeling/Decomposition Strategy in the MINLP Optimization of Process Flowsheets with Implicit Models. Ind. Eng. Chem. Res. 1996; 35:2065-2070.

Petrides DP, Calandris J, Cooney CL. Bioprocess optimization via CAPD and simulation for prodcut commercialization. Genet. Eng. New. 1996; 16:24-40.

Koulouris A, Calandranis J, Petrides DP. Throughput Analysis and Debottlenecking of Integrated Batch Chemical Processes. Computers and Chemical Engineering 2000; 24:13871394. 
Mendez CA, Cerda J, Grossmann IE, Harjunkoski I, Fahl M. State-of-the-art review of optimization methods for shrt-term scheduling of batch processes. Computers and Chemical Engineering 2006; 30:913-946.

Petrides DP, Papavasileiou V, Kolouris A. Siletti C. Optimize manufacturing of pharmaceutical products with process simulation and production. Chemical Engineering Research and Design 2006; 85:1086-1097.

Pfefferle W, Moeckel B, Bathe B, Marx A. Biotechnological manufacture of Lysine Adv. Biochem. Eng. Biotechnol 2003; 79:59-112.

Pistikopoulos EN, Sakizlis V, Perkins JD. Recent advances in optimization-based simultaneous process and control design. Computers and Chemical Engineering 2010; 28:2069-2086

Pozo C. Sorribas A. Vilaprinyo E. Guillén-Gosálbez G. Jiménez L. Alves R. Optimization and evolution in metabolic pathways: global optimization techniques in Generalized Mass Action models. Journal of biotechnology 2010; 149:141-153

Powell MJD. A Fast Algorithm for Nonlinearly Constrained Optimization Calculations. Numerical Analysis. Springer Verlag; 1978.

Raghunathan AU, RPérez-Correa JR, Biegler LT. Data Reconciliation and Parameter Estimation in Flux-Balance Analysis. Biotechnology and Bioengineering 2003; 84:700-709.

Shampine LF. Numerical Solution of Ordinary Differential Equations. Chapman and Hall, New York; 1994.

Stevens J, Blinder T. Porcess for making granular L-lysine. US Patent US 005990 350A; 1999.

Sarkar D, Modak JM. Pareto-optimal solutions for multi-objective optimization of fed-batch bioreactors using nondominated sorting genetic algorithms. Chemcial Engineering Science $2005 ; 60: 481-492$ 
Terrazas-Moreno S, Flores-Tlacuahuac A, Grossman IE. Simultaneous Cyclic Scheduling and Optimal Control of Polymerization Reactors. AICHE Journal 2007; 53:2301-2315

Viswanathan J, Grossman IE. A combined penalty function and outer approximation method for MINLP optimization. Computers and Chemical Engineering 1990; 14:769-782

Wong VVT, Oh SKW, Kuek KH. Design, simulation and optimization of a large scale monoclonal antibody production plant. Pharmaceutical Engineering 2004; 24:24-60 


\section{NOMENCLATURE}

$\begin{array}{ll}\text { Abbreviatures } & \\ \text { CF } & \text { centrifuge } \\ \text { COM } & \text { component Object Module } \\ \text { DAEs } & \text { differential-algebraic equations } \\ \text { MF } & \text { microfilter } \\ \text { MIDO } & \text { mixed-integer dynamic optimization } \\ \text { MILP } & \text { mixed-integer linear programming } \\ \text { MINLP } & \text { mixed-integer non-linear programming } \\ \text { NLP } & \text { non-linear programming } \\ \text { ODE } & \text { ordinary differential equation } \\ \text { RVF } & \text { rotary vacuum filter } \\ \text { SQP } & \text { successive quadratic programming } \\ \text { STY } & \text { space time yield }(\mathrm{g} / \mathrm{L} \cdot \mathrm{h}) \\ Y_{\text {oa }} & \text { overall yield (g/g) }\end{array}$

\section{Indices}

$\begin{array}{ll}\text { a } & \text { algebraic } \\ \text { d } & \text { differential } \\ \text { i } & \text { final } \\ \text { m } & \text { intermedium } \\ \text { n } & \text { type unit selected } \\ \mathrm{k} & \text { units in parallel } \\ \mathrm{p} & \text { iterations } \\ \mathrm{q} & \text { equality } \\ 0 & \text { inequality } \\ & \text { intial }\end{array}$

Variables 


$\begin{array}{ll}K_{M} & \text { substrate concentration at half max. rate }(\mathrm{g} / \mathrm{l}) \\ \text { NPV } & \text { Net Present Value }(\$) \\ \mathrm{S} & \text { Substrate concentration }(\mathrm{g} / \mathrm{l}) \\ \mathrm{STY} & \text { Space-time yield }(\mathrm{g} / \mathrm{l} \cdot \mathrm{h}) \\ \mathrm{VVM} & \text { Volume of air per volume of liquid per min } \\ Y_{\text {oa }} & \text { Overall-yield }(\mathrm{g} / \mathrm{g}) \\ \mu & \text { specific growth rate }(\mathrm{g} / \mathrm{l} \cdot \mathrm{h}) \\ \mu_{\text {max }} & \text { maximum specific growth rate }(\mathrm{g} / \mathrm{l} \cdot \mathrm{h})\end{array}$

\section{Bioreaction parameters}

$\begin{array}{ll}c_{L} & \text { oxygen concentration }(\mathrm{g} / \mathrm{L}) \\ c_{P} & \text { product concentration }(\mathrm{g} / \mathrm{L}) \\ c_{S} & \text { substrate concentration }(\mathrm{g} / \mathrm{L}) \\ c_{S F} & \text { substrate concentration in the feed }(\mathrm{g} / \mathrm{L}) \\ c_{s I N} & \text { initial substrate concentration }(\mathrm{g} / \mathrm{L}) \\ c_{T h r} & \text { threonine concentration }(\mathrm{g} / \mathrm{L}) \\ c_{x} & \text { biomass concentration }(\mathrm{g} / \mathrm{L}) \\ \mathrm{F} & \text { rate of feed }(\text { feed rate })(\mathrm{L} / \mathrm{h}) \text { or }(\mathrm{m} 3 / \mathrm{h}) \\ K_{L} a & \text { specific mass transfer coefficient }(1 / \mathrm{h}) \\ K_{I P} & \text { product inhibition constant }(\mathrm{g} / \mathrm{L}) \\ K_{I T h r} & \text { threonine inhibition constant }(\mathrm{g} / \mathrm{L}) \\ K_{O} & \text { substrate oxygen affinity constant }(\mathrm{g} / \mathrm{L}) \\ K_{P S} & \text { product affinity constant }(\mathrm{g} / \mathrm{L}) \\ K_{S} & \text { substrate carbon source affinity constant }(\mathrm{g} / \mathrm{L}) \\ K_{T h r} & \text { substrate threonine affinity constant }(\mathrm{g} / \mathrm{L}) \\ L_{O 2} & \text { oxygen solubility }(\text { mol/L/bar }) \\ \text { mo } & \text { specific oxygen consumption for maintenance }(\mathrm{g} / \mathrm{L}) \\ \text { ms } & \text { specific substrate consumption for maintenance }(\mathrm{g} / \mathrm{L}) \\ & \end{array}$




\begin{tabular}{|c|c|}
\hline OTR & oxygen transfer rate $(\mathrm{mol} / \mathrm{L} \cdot \mathrm{h})$ \\
\hline$P_{R}$ & reactor pressure (bar) \\
\hline$r_{p}$ & rate of lysine production $(\mathrm{g} / \mathrm{L} \cdot \mathrm{h})$ \\
\hline STY & space time yield $(\mathrm{g} / \mathrm{L} \cdot \mathrm{h})$ \\
\hline $\mathrm{t}$ & time $(h)$ \\
\hline $\mathrm{V}$ & fermenter filling volume (m3) \\
\hline$y_{l}$ & mole fraction of oxygen in the liquid phase $(\mathrm{mol} / \mathrm{mol})$ \\
\hline$y_{o 2}$ & mole fraction of oxygen in the gas phase $(\mathrm{mol} / \mathrm{mol})$ \\
\hline$Y_{o a}$ & overall yield (g/g) \\
\hline$Y_{P / O}$ & product yield per amount of oxygen (g/g) \\
\hline$Y_{P / S}$ & product yield per amount of substrate (g/g) \\
\hline$Y_{x / s}$ & biomass yield per amount of substrate (g/g) \\
\hline$Y_{x / o}$ & biomass yield per amount of oxygen (g/g) \\
\hline$Y_{x / T h r}$ & biomass yield per amount of threonine (g/g) \\
\hline$a_{p}$ & growth-associated coefficient for product synthesis (g/g) \\
\hline ßp & non-growth-associated coefficient for product synthesis $(\mathrm{g} / \mathrm{g} \cdot \mathrm{h})$ \\
\hline$\mu$ & specific growth rate $(1 / \mathrm{h})$ \\
\hline$\mu_{\max }$ & maximum specific growth rate $(1 / \mathrm{h})$ \\
\hline
\end{tabular}




\section{A Biochemical reaction model for a fed-batch reactor to produce L-lysine}

Mass balance for glucose $\frac{d c_{s}}{d t}=-\frac{1}{Y_{X / S}} \cdot \mu \cdot c_{x}-\frac{1}{Y_{P / S}} \cdot r_{p} \cdot c_{x}-m_{s} \cdot c_{x}+\frac{F}{V}\left(c_{S F}-c s\right)$

Mass balance for oxygen $\frac{d c_{L}}{d t}=-\frac{1}{Y_{X / O}} \cdot \mu \cdot c_{x}-\frac{1}{Y_{P / O}} \cdot r_{p} \cdot c_{x}-m_{s} \cdot c_{x}+O T R$

Mass balance for threonine $\frac{d c_{T h r}}{d t}=-\frac{1}{Y_{X / T h r}} \cdot \mu \cdot c_{x}-\frac{F}{V}\left(c_{T h r}\right)$

Mass balance for biomass $\frac{d c_{x}}{d t}=\mu \cdot c_{x}-\frac{F}{V} \cdot c_{x}$

Mass balance for lysine $\frac{d c_{P}}{d t}=r_{P} \cdot c_{x}-\frac{F}{V} \cdot c_{P}$

Mass balance for the fermenter volume $\frac{d V}{d t}=F$

Kinetic model for oxygen transfer $O T R=k_{L} a \cdot L_{O_{2}} \cdot p_{R} \cdot\left(y_{O_{2}}-y_{L}\right)$

Kinetic model for growth $\mu=\mu_{\max } \cdot \frac{c_{s}}{c_{s}+K_{s}} \cdot \frac{c_{L}}{c_{L}+K_{O}} \cdot \frac{c_{L}}{c_{L}+K_{T} h r}$

Kinetic model for lysine formation $r_{P}=\left(\alpha_{P} \cdot \mu+\beta_{P}\right) \cdot \frac{c_{s}}{c_{s}+K_{P S}} \cdot \frac{c_{L}}{c_{L}+K_{O}} \cdot \frac{K_{I T h r}}{c_{T h r}+K_{I T h r}} \cdot \frac{K_{I P}}{c_{P}+K_{I P}}$

Overall yield $Y_{o a}=\frac{c_{p}}{c_{S_{I N}}}$

Space-time yield $S T Y=\frac{c_{P}}{t}$ 


\section{List of Tables}

1 Progress of iterations of MIDO algorithm in the optimization of component

A production plant . . . . . . . . . . . . . . . . . . . . . . 33

$2 \quad$ Results of the optimization of component A production plant . . . . . . . . . 34

3 Progress of iterations of MIDO algorithm in the optimization of L-lysine pro-

duction plant . . . . . . . . . . . . . . . . . . . . . . . 35

4 Results of the optimization of L-lysine production plant . . . . . . . . . . . 36 
Table 1: Progress of iterations of MIDO algorithm in the optimization of component A production plant

\begin{tabular}{lccc}
\multicolumn{1}{c}{ Iteration Number } & NLP1 & MILP1 & NLP2 \\
\hline Discrete decisions & & & \\
Fermentors & 1 & 2 & 2 \\
Equipment separation phase & Decanter & Decanter & Decanter \\
Objective function & & & \\
$J^{k}[\$]$ & $2.18 \cdot 10^{7}$ & $2.79 \cdot 10^{7}$ & $1.90 \cdot 10^{7}$ \\
CPU time $[\mathrm{s}]$ & 5.59 & 0.15 & 6.87 \\
\hline
\end{tabular}


Table 2: Results of the optimization of component A production plant Initial Point Optimal Point

\begin{tabular}{lcc}
\hline Net present value [M $\$$ ] & 20,870 & 21,854 \\
Total capital investment [M $\$$ ] & 6.164 & 5.874 \\
Operating cost [M $\$ /$ year] & 2.962 & 2.758 \\
Production rate [kg A/year] & 2,025 & 2,025 \\
Unit production cost [\$/kg A] & 1.462 & 1.362 \\
Batch throughput [kg A/batch] & 5.84 & 6.16 \\
Recipe batch time [h] & 19.27 & 20.18 \\
Recipe cycle time [h] & 17.27 & 18.18 \\
Annual operating time [h] & 5,994 & 5,984 \\
Number of batches per year & 347 & 329 \\
Substrate concentration [g/l] & 53.47 & 80.00 \\
Reaction time [h] & 15.27 & 16.18 \\
Fermentors & 1 & 1 \\
Fermentor volume [l] & 7675.89 & 5454.16 \\
Separator & Decanter & Decanter \\
Volume separator [l] & 97.32 & 56.47 \\
\hline
\end{tabular}


Table 3: Progress of iterations of MIDO algorithm in the optimization of L-lysine production plant

\begin{tabular}{lccccccc} 
Iteration Number & NLP1 & MILP1 & NLP2 & MILP2 & NLP3 & MILP3 & NLP4 \\
\hline Discrete decisions & & & & & & & \\
Fermentors & 1 & 2 & 2 & 2 & 2 & 3 & 3 \\
Equipment separation phase & $\mathrm{MF}$ & $\mathrm{MF}$ & $\mathrm{MF}$ & $\mathrm{RVF}$ & $\mathrm{RVF}$ & $\mathrm{RVF}$ & $\mathrm{RVF}$ \\
Objective function & & & & & & & \\
$J^{k}[\$]$ & $7.01 \cdot 10^{7}$ & $2.00 \cdot 10^{8}$ & $1.66 \cdot 10^{8}$ & $1.85 \cdot 10^{8}$ & $1.95 \cdot 10^{8}$ & $2.26 \cdot 10^{8}$ & $1.82 \cdot 10^{8}$ \\
CPU time [s] & 55.34 & 0.21 & 44.04 & 0.17 & 41.20 & 0.19 & 53.75 \\
\hline
\end{tabular}


Table 4: Results of the optimization of L-lysine production plant

Base Case Initial Point Optimal Point

\begin{tabular}{lccc}
\hline Net present value [M\$] & 172,003 & 59,276 & 195,688 \\
Total capital investment [M\$] & 101.766 & 55.369 & 79.885 \\
Operating cost [M\$/year] & 10.631 & 4.854 & 8.830 \\
Production rate [tones L-lysine/year] & 6,202 & 2,611 & 6,202 \\
Unit Production cost [\$/kg L-lysine] & 1.71 & 1.86 & 1.42 \\
Batch Throughput [tons L-lysine/batch] & 29.674 & 27.783 & 44.300 \\
Recipe Batch time [h] & 111.07 & 110.46 & 137.67 \\
Recipe Cycle time [h] & 37.51 & 83.51 & 55.81 \\
Number of batches per year & 209 & 94 & 140 \\
Concentration Threonine [g/l] & 1.62 & 1.62 & 1.92 \\
Concentration Glucose [g/l] & 48.72 & 48.72 & 94.61 \\
Initial Volume Ferment [m $\left.{ }^{3}\right]$ & 310.34 & 310.34 & 282.77 \\
Reaction time [h] & 71.01 & 71.01 & 97.16 \\
Fermentors & 3 & 1 & 2 \\
Space-time yield $[\mathrm{g} / \mathrm{l} \cdot \mathrm{h}]$ & 1.022 & 1.022 & 1.103 \\
Overall yield $[\mathrm{g} / \mathrm{g}]$ & 0.299 & 0.299 & 0.316 \\
Separator & $\mathrm{RVF}$ & $\mathrm{MF}$ & $\mathrm{RVF}$ \\
\hline
\end{tabular}




\section{List of Figures}

1 Flowchart of the proposed algorithm . . . . . . . . . . . . . . . 38

$2 \quad$ Main steps in the resolution of the NLP sub-problem . . . . . . . . . . . . . 39

3 Process flow diagram of a typical ferementation process . . . . . . . . . . . . 40

4 Preliminary analysis of the decision variables in the case study 1 . . . . . . . 41

5 Completed reaction time (i.e., reaction time for which concentration of sub-

strate is zero)versus substrate concentration . . . . . . . . . . . . . . . . . . 42

$6 \quad$ L-lysine production plant (adapted from Heinzle et al.,2006) . . . . . . . . . 43

7 Preliminary analysis of the decision variables in case study 2 . . . . . . . . . 44

8 Preliminary analysis of the decision variables in case study 2 . . . . . . . 45 


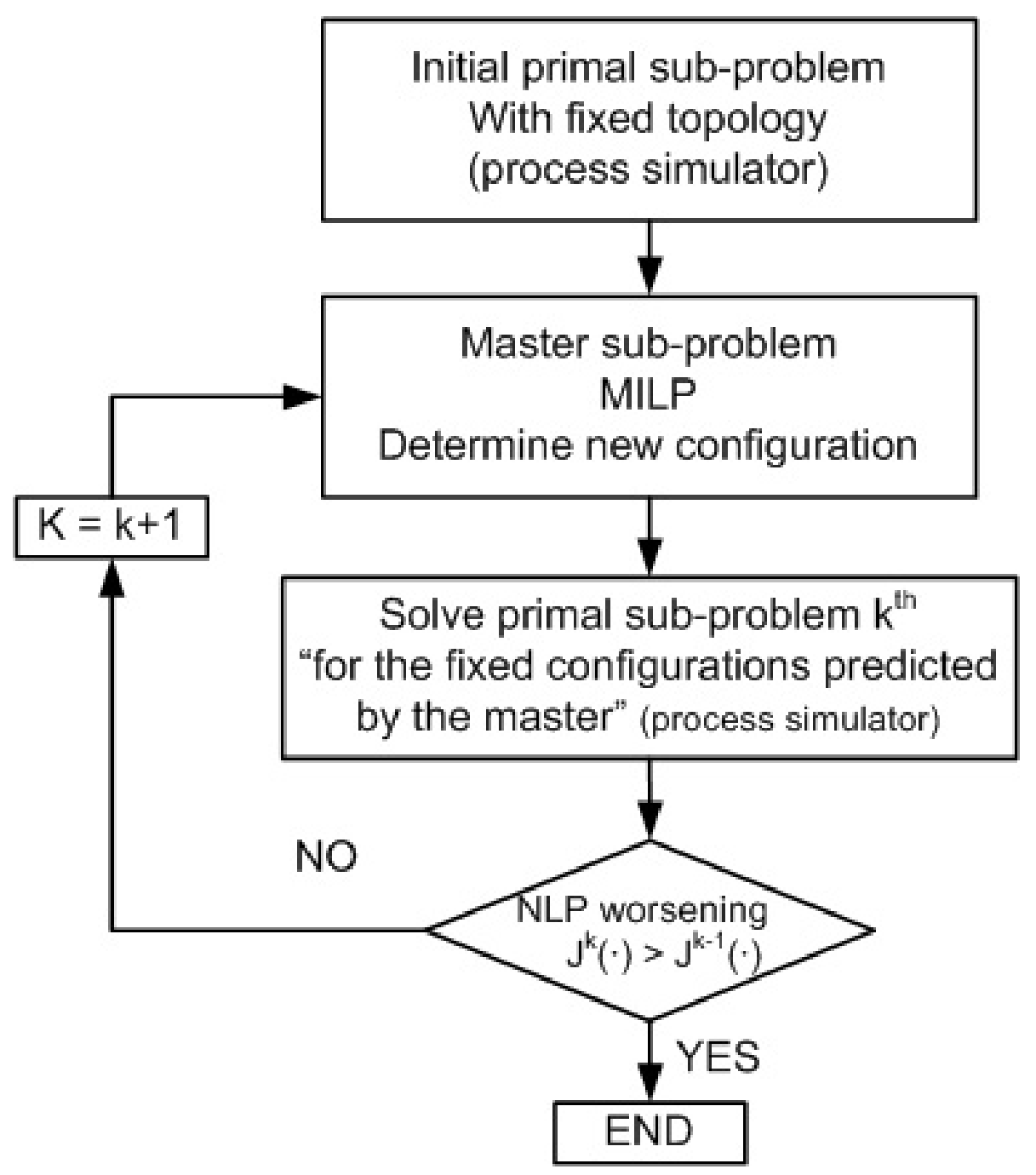

Figure 1: Flowchart of the proposed algorithm 


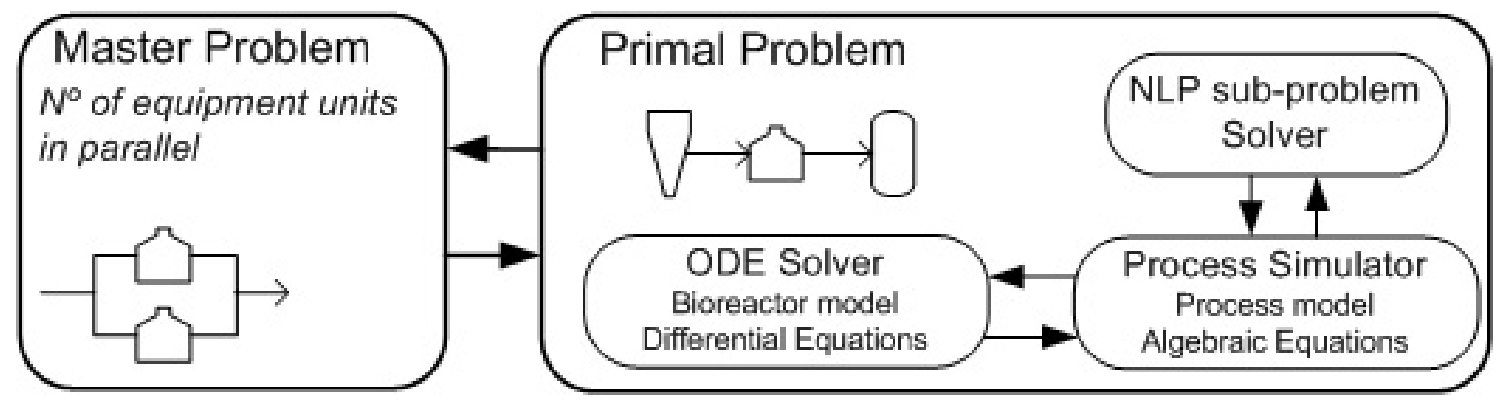

Figure 2: Main steps in the resolution of the NLP sub-problem 


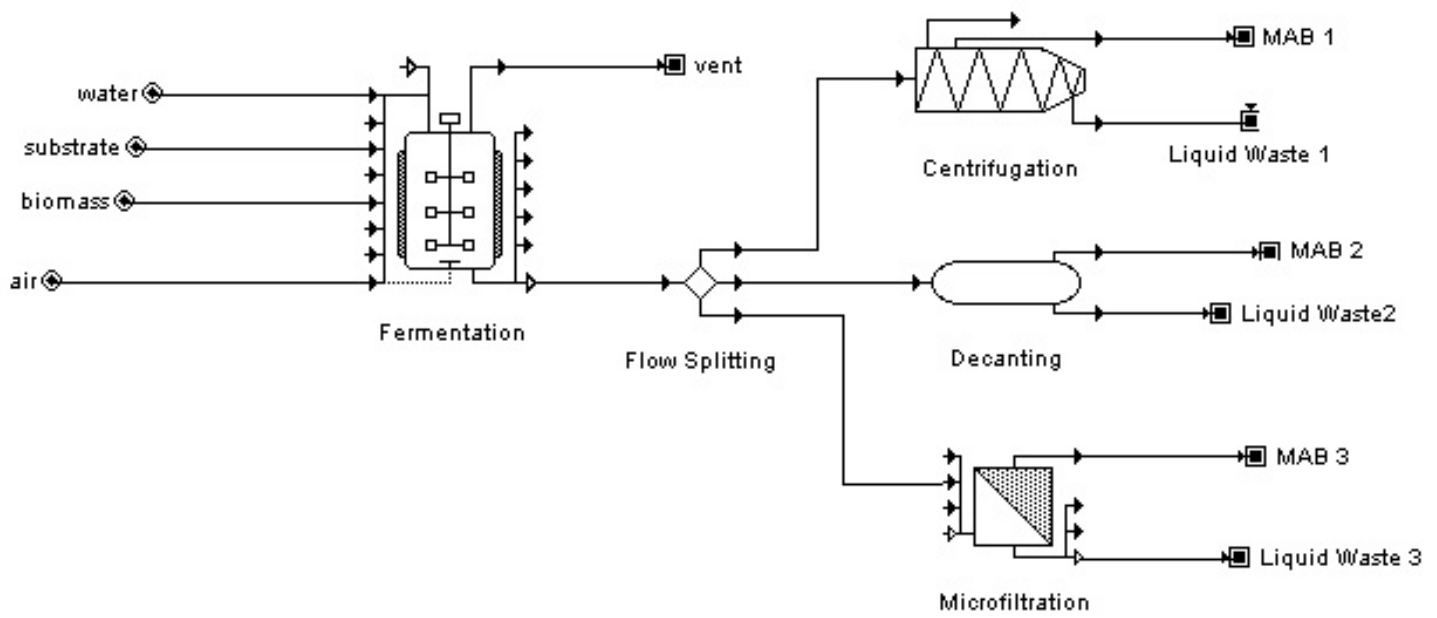

Figure 3: Process flow diagram of a typical ferementation process 


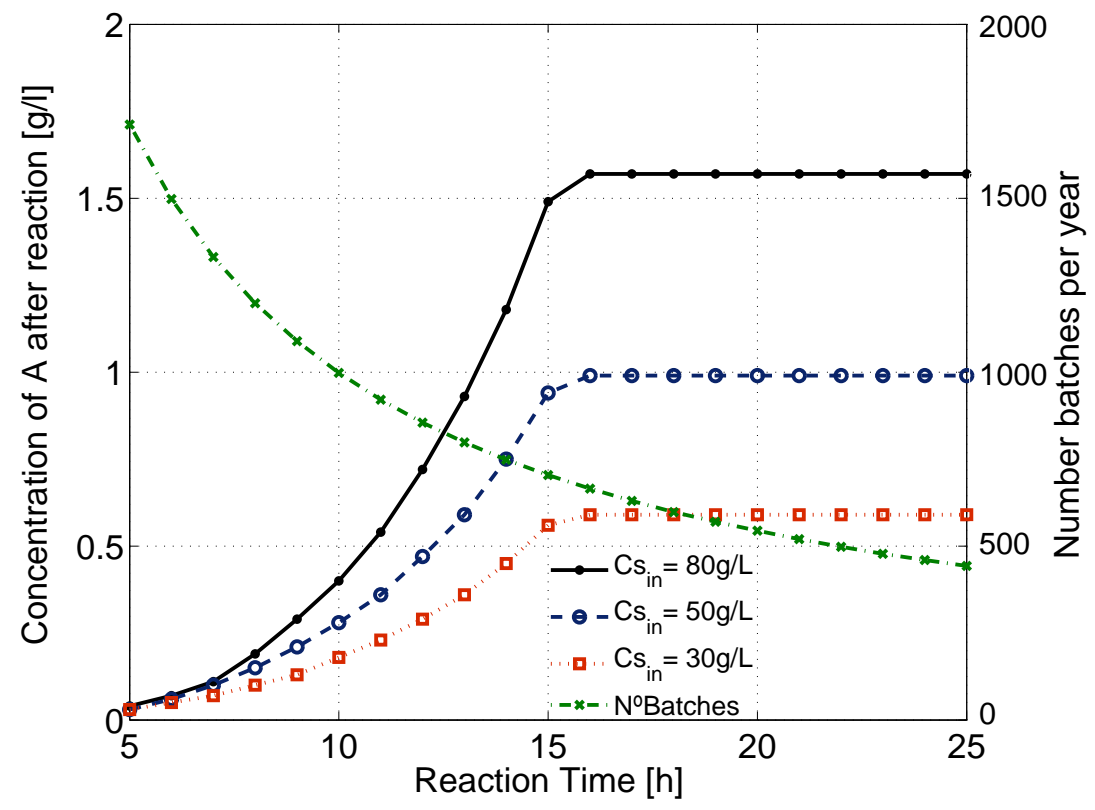

(a) Concentration of $\mathrm{A}$ and number of batches versus reaction time for a fixed demand of $2025 \mathrm{~kg} / \mathrm{yr}$

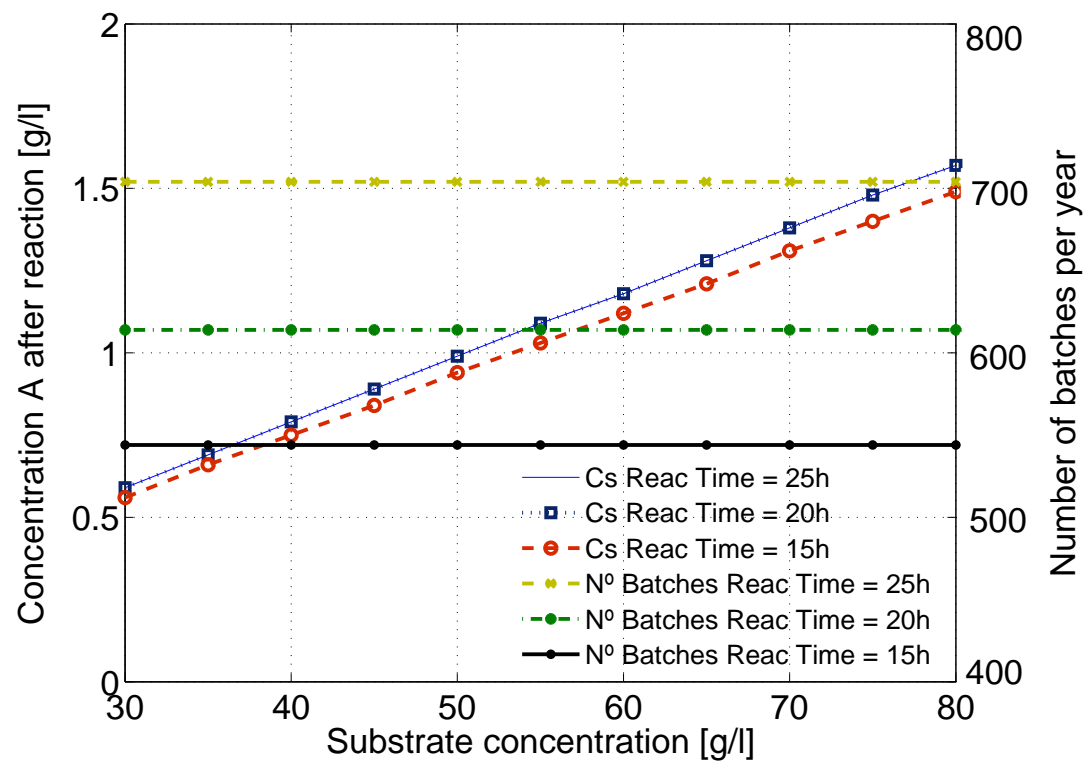

(b) Concentration of $\mathrm{A}$ and number of batches versus initial substrate concentration for a fixed demand of $2025 \mathrm{~kg} / \mathrm{yr}$

Figure 4: Preliminary analysis of the decision variables in the case study 1 


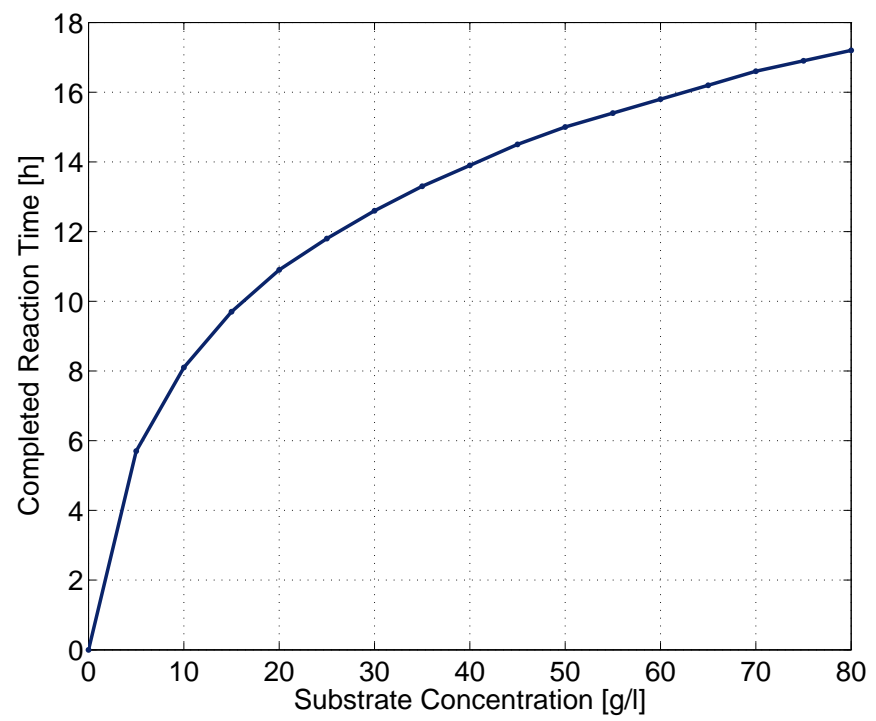

Figure 5: Completed reaction time (i.e., reaction time for which concentration of substrate is zero)versus substrate concentration 


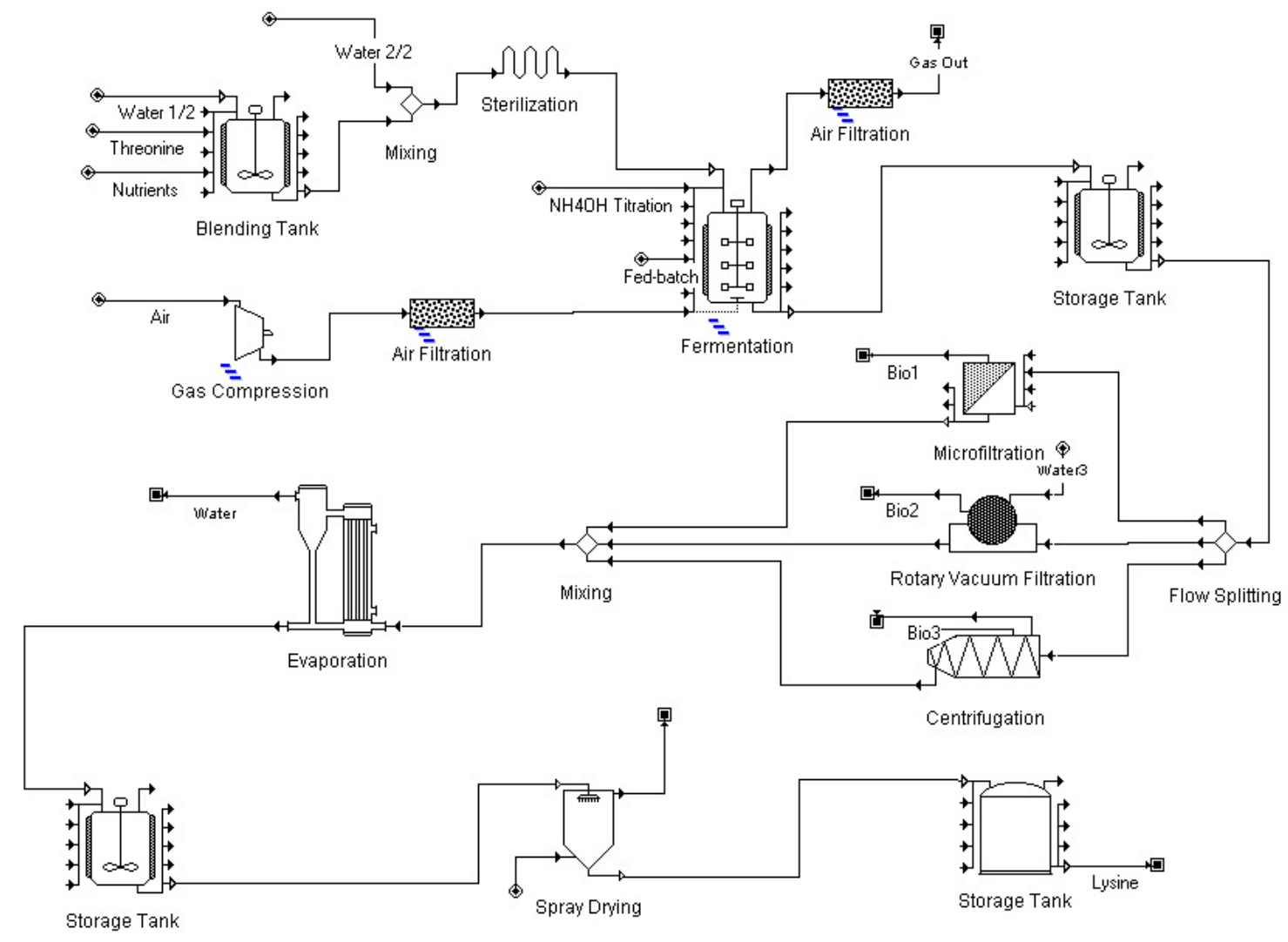

Figure 6: L-lysine production plant (adapted from Heinzle et al.,2006) 


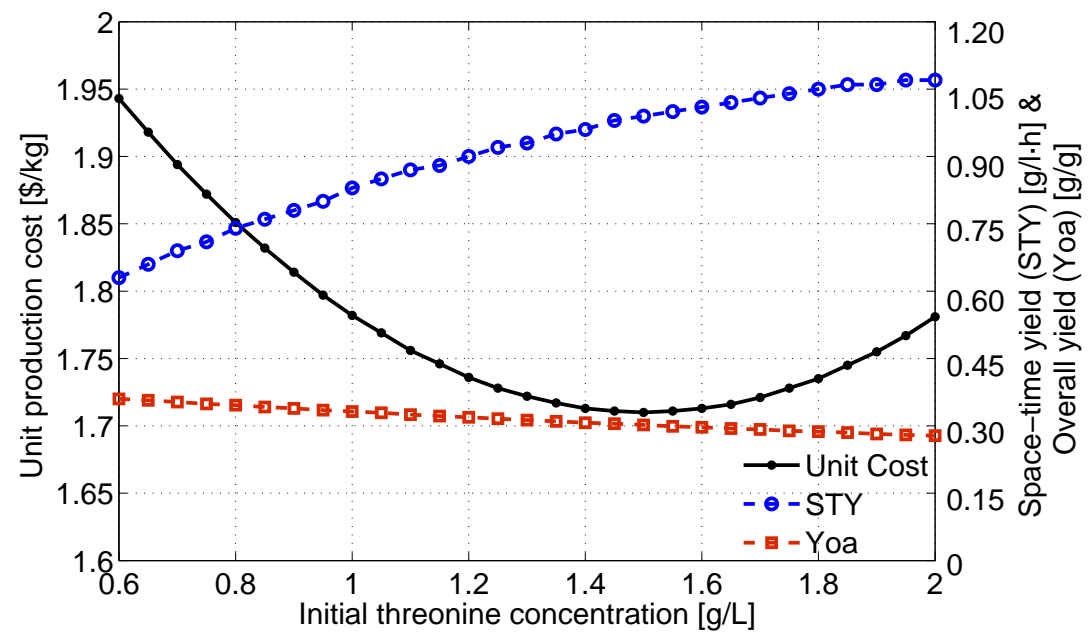

(a) Relationship between the unit production cost, $S T Y$ and $Y_{o a}$ and the initial threonine concentration $\left(C_{T h r}\right)$, maintaining the rest of the decision variables constant

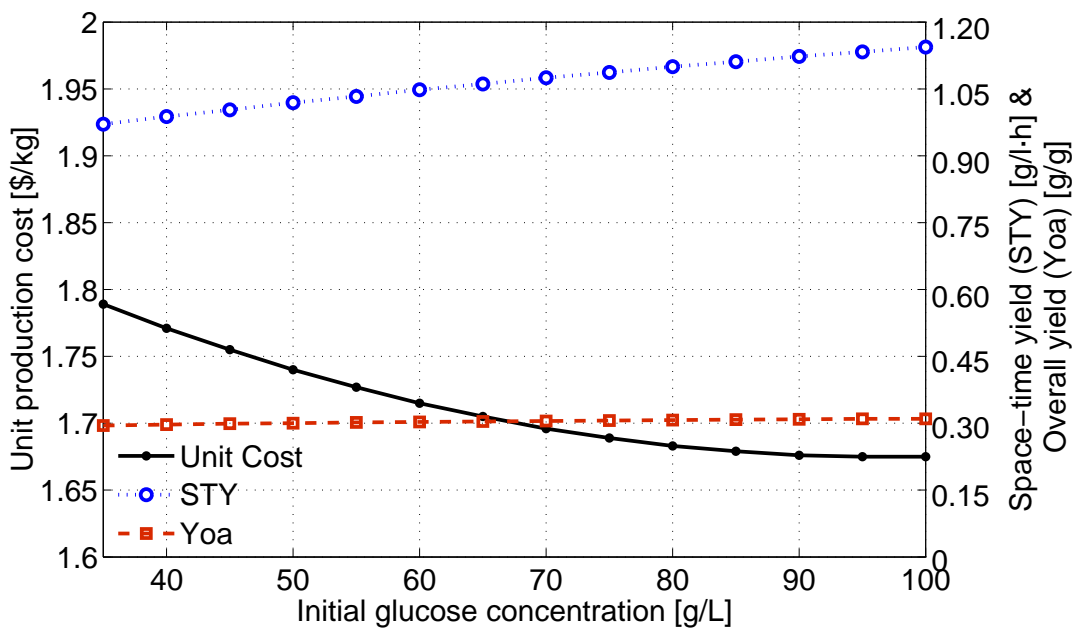

(b) Relationship between the unit production cost, $S T Y$ and $Y_{o a}$ and the initial glucose concentration $\left(C s_{i n}\right)$, maintaining the rest of the decision variables constant

Figure 7: Preliminary analysis of the decision variables in case study 2 


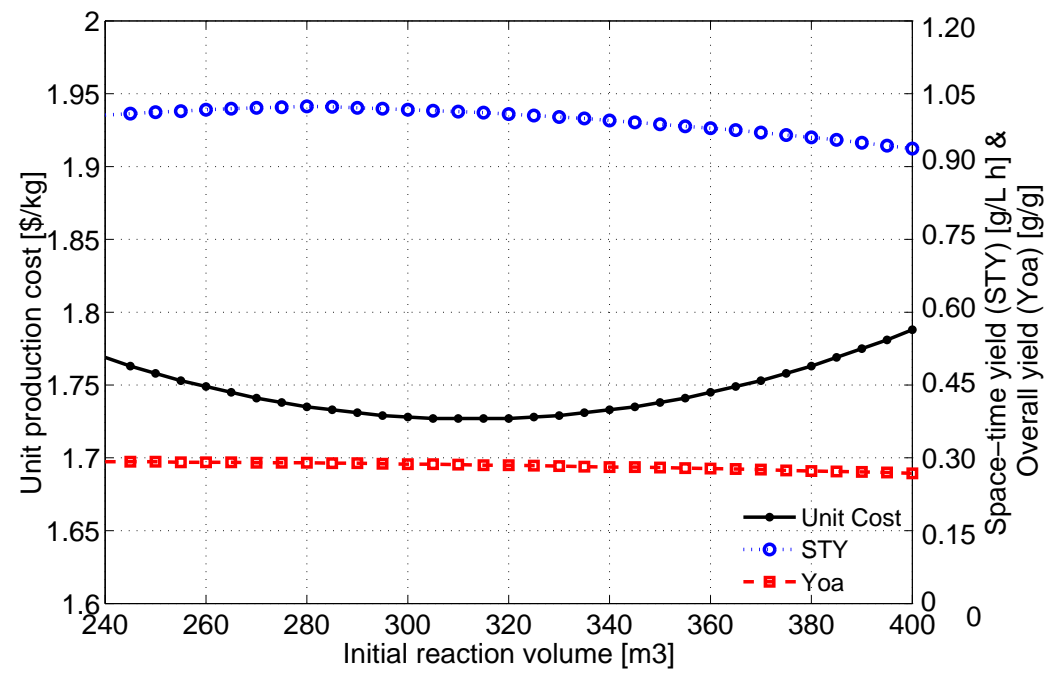

(a) Relationship between the unit production cost, STY and $Y_{o a}$ and the initial reactor volume, maintaining the rest of the decision variables constant

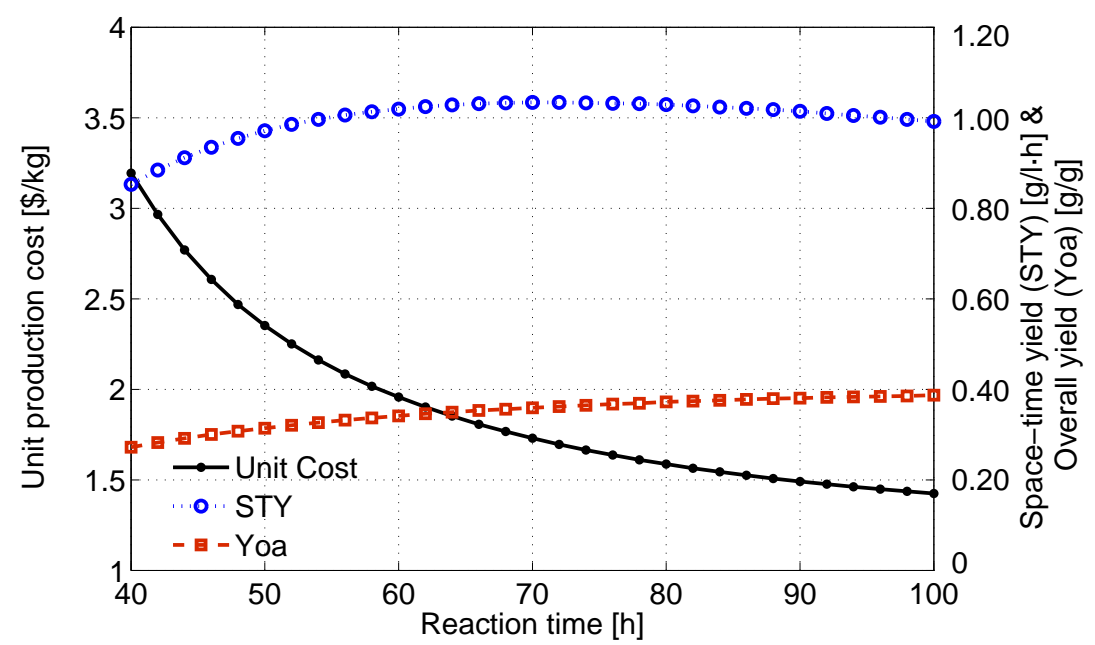

(b) Relationship between the unit production cost, $S T Y$ and $Y_{o a}$ and the initial reaction time, maintaining the rest of the decision variables constant

Figure 8: Preliminary analysis of the decision variables in case study 2 